\title{
Status of COMPASS RICH-1 Upgrade with MPGD-based Photon Detectors
}

\author{
M. Alexeev ${ }^{1, a}$, R Birsa ${ }^{2}$, F. Bradamante ${ }^{2,3}$, A. Bressan ${ }^{2,3}$, M. Chiosso $^{1,4}$, P. Cilibertiं ${ }^{2,3}$, S. Dalla Torre ${ }^{2}$, S. Dasgupta 2,3 , \\ O. Denisov ${ }^{1}$, M. Finger ${ }^{5,6}$, M. Finger Jr ${ }^{5,6}$, H. Fishcher ${ }^{7}$, B. Gobbo ${ }^{2}$, M. Gregori ${ }^{2}$, G. Hamar ${ }^{2}$, F. Herrmann ${ }^{7}$, K. \\ Königsmann $^{7}$, S. Levorato ${ }^{2}$, A. Maggiora ${ }^{1}$, N. Makke ${ }^{2,3}$, A. Martin ${ }^{2,3}$, G. Menon ${ }^{2}$, J. Novy ${ }^{5,6}$, D. Panzieri', ${ }^{1,8}$ F. \\ A. Pereira ${ }^{9}$, C. A. Santos ${ }^{2}$, G. Sbrizzai ${ }^{2,3}$, P. Schiavon ${ }^{2,3}$, S. Schopferer ${ }^{7}$, M. Slunechka ${ }^{5,6}$, K. Steiger ${ }^{2,10, b}$, L. \\ Steiger ${ }^{2,10, b}$, M. Sulc ${ }^{10}$, F. Tessarotto ${ }^{2, c}$, and J. F. C. A. Veloso ${ }^{9}$
}

${ }^{1}$ INFN Sezione di Torino, Torino, Italy

${ }^{2}$ INFN Sezione di Trieste, Trieste, Italy

${ }^{3}$ University of Trieste, Trieste, Italy

${ }^{4}$ University of Torino, Torino, Italy

${ }^{5}$ Charles University, Prague, Czech Republic

${ }^{6}$ JINR, Dubna, Russia

${ }^{7}$ Universität Freiburg, Physikalisches Institut, Freiburg, Germany

${ }^{8}$ University of East Piemonte, Alessandria, Italy

${ }^{9}$ Physics Department, University of Aveiro, Aveiro, Portugal

${ }^{10}$ Technical University of Liberec, Liberec, Czech Republic

\begin{abstract}
A Set of new MPGD-based Photon Detectors is being built for the upgrade of COMPASS RICH1. The detectors cover a total active area of $1.4 \mathrm{~m}^{2}$ and are based on a hybrid architecture consisting of two THGEM layers and a Micromegas. A CsI film on one THGEM acts as a reflective photocathode. The characteristics of the detector, the production of the components and their validation tests are described in detail.
\end{abstract}

\section{The COMPASS RICH-1 Upgrade}

The COMPASS Experiment at CERN SPS has recently started a new set of measurements [1] imposing strict requirements in terms of rate capability, efficiency, and stability of the detector performance: several parts of the apparatus are being upgraded to meet these requirements, including the COMPASS RICH-1 detector [2], which provides $\pi$-K separation from 3 to $55 \mathrm{GeV} / c$ over $\pm 200 \mathrm{mrad}$ angular acceptance, at high rates.

COMPASS RICH-1 (Fig. 1, left) is a Ring Imaging Cherenkov counter with a $3 \mathrm{~m}$ long gaseous $\mathrm{C}_{4} \mathrm{~F}_{10}$ radiator, a $21 \mathrm{~m}^{2}$ large focusing VUV mirror surface and Photon Detectors (PDs) covering a total active area of $5.5 \mathrm{~m}^{2}$ with two technologies: Multi-Anode PMTs coupled to individual fused silica lens telescopes in the central region (25\% of the surface) and MWPCs equipped with CsIcoated photocathodes in the remaining surface.

In spite of their good performance, MWPC-based PDs present intrinsic limitations: aging (decrease of quantum efficiency) after a few $\mathrm{mC} / \mathrm{cm}^{2}$ charge collection, feedback pulses with a rate increasing at large gain values, long recovery time after occasional discharge in the de-

\footnotetext{
${ }^{a}$ On leave from JINR, Dubna, Russia.

${ }^{b}$ Their present address is Technical University of Liberec, Liberec, Czech Republic and Institute of Plasma Physics, Academy of Sciences of the Czech Republic, Turnov, Czech Republic.

${ }^{\mathrm{c} C}$ Corresponding author; email:fulvio.tessarotto@ts.infn.it
}

tector and long signal formation time. The MWPCs have to be operated at low gain and present a non-negligible detector memory and dead time. Among the eight MWPC's of COMPASS RICH-1 four, located above and below the centre of the detector show particularly critical performance [3]. The present upgrade aims at replacing them with novel MPGD-based PDs, developed in a seven yearlong dedicated R\&D programme [4]: the resulting detector architecture is a Hybrid MPGD including two THick GEM (THGEM) multiplication stages followed by a Micromegas.

The main technical characteristics and the production process of the MPGD-based PDs for COMPASS RICH-1 upgrade are described in this article.

\section{The THGEM production and quality assessment}

Each one of the four new chambers (Fig. 1, right) will host one of the existing $600 \times 600 \mathrm{~mm}^{2}$ panel of Multi-Anode PMTs and lens telescopes together with a new MPGDbased PD consisting of two identical hybrid modules covering about $600 \times 300 \mathrm{~mm}^{2}$, arranged side by side.

The basic structure of the hybrid module (Fig. 2) consists of two layers of THGEMs, one Micromegas, and two planes of wires. The top layer of the first THGEM (as seen 

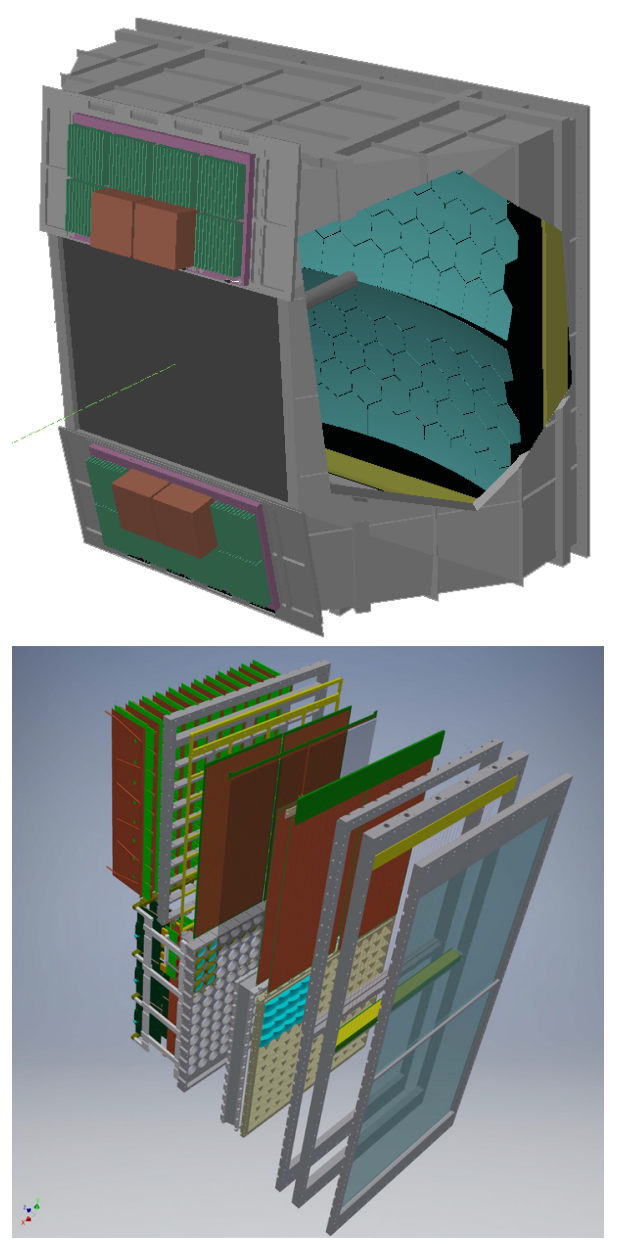

Figure 1. Artistic view of the COMPASS RICH-1 detector (left) and exploded view of the new PDs (right)

from inside the RICH vessel) is coated with a CsI film and acts as a reflective photocathode for VUV photons.

The THGEM PCBs are all identical: they have an average thickness of $470 \mu \mathrm{m}$ (400 $\mu \mathrm{m}$ of fiberglass and $2 \times 35$ $\mu \mathrm{m} \mathrm{Cu}$ ), a length of $581 \mathrm{~mm}$ and a with of $287 \mathrm{~mm}$. They have a regular hexagonal array of holes of $400 \mu \mathrm{m}$ diameter, with a pitch of $800 \mu \mathrm{m}$. The holes are produced by mechanical drilling and have no rim. The holes of the external border lines have $500 \mu \mathrm{m}$ diameter, to avoid problems related to the asymmetric field configuration at the electrode edges. On both top and bottom face the active area is divided in 12 sectors which are $564 \mathrm{~mm}$ long and 23.3 $\mathrm{mm}$ wide (the two external ones are $17.9 \mathrm{~mm}$ wide), with a separation of $0.7 \mathrm{~mm}$ between neighboring sectors. Electrical connections are individually provided to each sector on one side of the THGEM. A set of 24 holes, used as fixation points, guarantees the correct planar positioning of the THGEM.

The PCB raw material (halogen-free EM 370-5 from Elite Material Co, Ltd.) has been carefully selected before the THGEM production phase: 50 foils (of $1245 \mathrm{~mm}$ $\times 1095 \mathrm{~mm}$ ) have been purchased, reduced to $800 \mathrm{~mm} \times$ $800 \mathrm{~mm}$ by cutting out the borders which usually present larger local thickness variations and mapped by means of

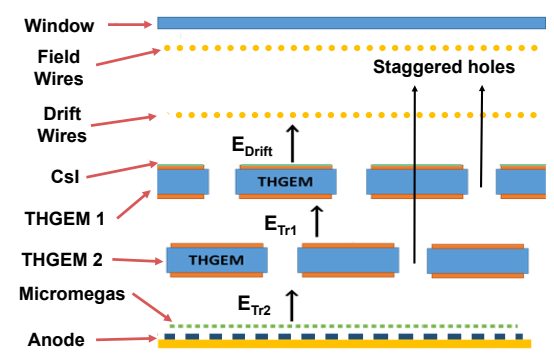

Figure 2. Schematic section of the hybrid module (not in scale).

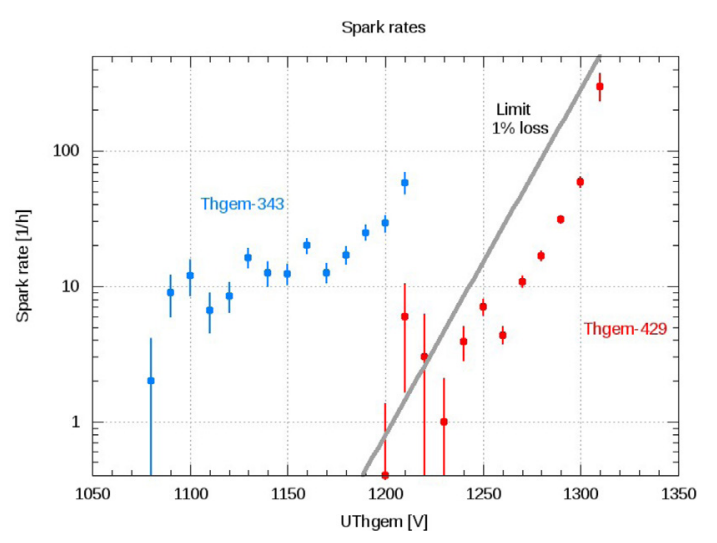

Figure 3. Rate of discharges in THGEMs as function of the bias voltage in $\mathrm{Ar} / \mathrm{CO}_{2}$ 70/30 gas mixture.

a Mitutoyo EURO CA776 coordinate measuring machine hosted in a thermally stabilized room. The PCB foil was laying on the reference surface of the coordinate measuring machine, kept flat by under-pressure while its top surface was mapped in a square pattern of points at $20 \mathrm{~mm}$ steps; the measurement was than repeated after reversing the foil and good consistency between the two measurements was obtained. The reference surface was regularly re-measured and the point by point difference between reference surface and foil measurement provided the local foil thickness values. A typical thickness distribution for a good foil presents an average value of $472 \mu \mathrm{m}$ and a standard deviation of $2 \mu \mathrm{m}$. Each PCB foil can host two raw THGEM PCBs, and a thickness uniformity quality factor $\delta_{t h}$ is evaluated for each surface of the PCB corresponding to a raw THGEM PCB. The quality factor is defined as $\delta_{t h}=\left(t h_{\max }-t h_{\min }\right) / t h_{\text {min }}$ where $t h_{\text {max }}$ and $t h_{\text {min }}$ are the maximum and minimum of the measured thickness values in the active area of the THGEM. The best raw THGEM PCBs have $\delta_{t h} \leq 2 \%$, and out of a total of 100 raw THGEM PCBs, 49 have $\delta_{\text {th }}<3 \%, 38$ have $3 \% \leq \delta_{\text {th }}<$ $4 \%$ and 13 have $\delta_{t h} \geq 4 \%$.

After the mapping and selection of the raw material 42 THGEMs have been produced. Individual foil labels and orientation marks allow to use the database of the local thickness values in the later stages of the THGEM production, test and operation. The mechanical drilling of the THGEM holes has been performed by ELTOS S.p.A. (Arezzo, Italy) after the image transfer, development, etching and dry film stripping operations have been completed. 
Up to 6 THGEMs can be drilled in parallel by using a multi-spindle machine (Posalux 6000-LZ). To guarantee a good quality of the hole surface no more than 1000 holes are drilled by a single tool. No electroplating process is applied and electrical continuity between top and bottom paths for the voltage bias is provided by copper rivets where needed.

A specific smoothing and cleaning procedure has been developed [5] and is applied to the 42 THGEMs in the Trieste COMPASS Laboratory: it consists in a long, careful polishing operation using fine-grain pumice powder and a manually guided vibration-rotation machine, followed by cleaning using high pressure water and ultrasonic bath in a basic solution (Sonica PCB detergent with PH=11), distilled water rinsing and drying by blowing nitrogen and storing in a vacuum tank or nitrogen flushed box.

For the quality assessment tests the THGEM is mounted in a box equipped with a pad-segmented anode and a wire plane biased to provide a proper drift field; it is flushed with an $\mathrm{Ar} / \mathrm{CO}_{2} 70 / 30$ gas mixture at $10 \mathrm{l} / \mathrm{h}$ flow and connected to a remotely controlled power supply programmed to provide from each electrode current monitoring and discharge counting. Discharges are defined as monitored current values larger than $50 \mathrm{nA}$. The electrical stability test program increases the bias voltage in steps of $10 \mathrm{~V}$ until a few discharges are registered; the bias voltage is thus lowered and the test cycle restarts. Sparks are sometimes associated to discharges and allow to visually identify the location of the discharge. Simultaneous discharges often appear in neighboring sectors and also in distant sectors and even in different test boxes, suggesting cosmic shower triggered events. A discharge rate below 1 event per hour is considered acceptable and the maximum stable voltage is defined accordingly. If all sectors of the THGEM have a maximum stable voltage larger than 1200 $\mathrm{V}$ (gain $\approx 3 \times$ the operational gain) then the THGEM is validated for electrical stability. In Fig. 3, the rate of discharges for a typical validated THGEM (n. 429), and for a rejected one (n. 343) are plotted as function of the bias voltage. The line corresponding to the typical discharge rate for THGEMs at the validation threshold limit is shown in gray. The failure rate for this validation stage is about $30 \%$ but reapplying the polishing and cleaning procedure allows to recover some of the THGEMs which fail the first electrical stability test.

The gain uniformity of each THGEM has been measured for the first produced pieces by using a ${ }^{55} \mathrm{Fe} \mathrm{X}$-ray source sequentially positioned in 72 points and collecting the signal amplitude spectra from the the set of anodic pads (covering about $20 \mathrm{~cm}^{2}$ ) in front of the source position via a CREMAT CR-110 preamplifier, a CANBERRA AFT Research Amplifier model 2025 and an AMPTEK 8000A MCA. Typical standard deviation values range from $7 \%$ to $10 \%$ thanks to the strict thickness selection criteria applied for the raw THGEM PCBs.

For all THGEMs except the first ones a new procedure has been implemented for the gain uniformity measurement: the X-ray source is an AMPTEK Mini-X with Au target and a $\mathrm{Cu}$ filter, providing uniform illumination over

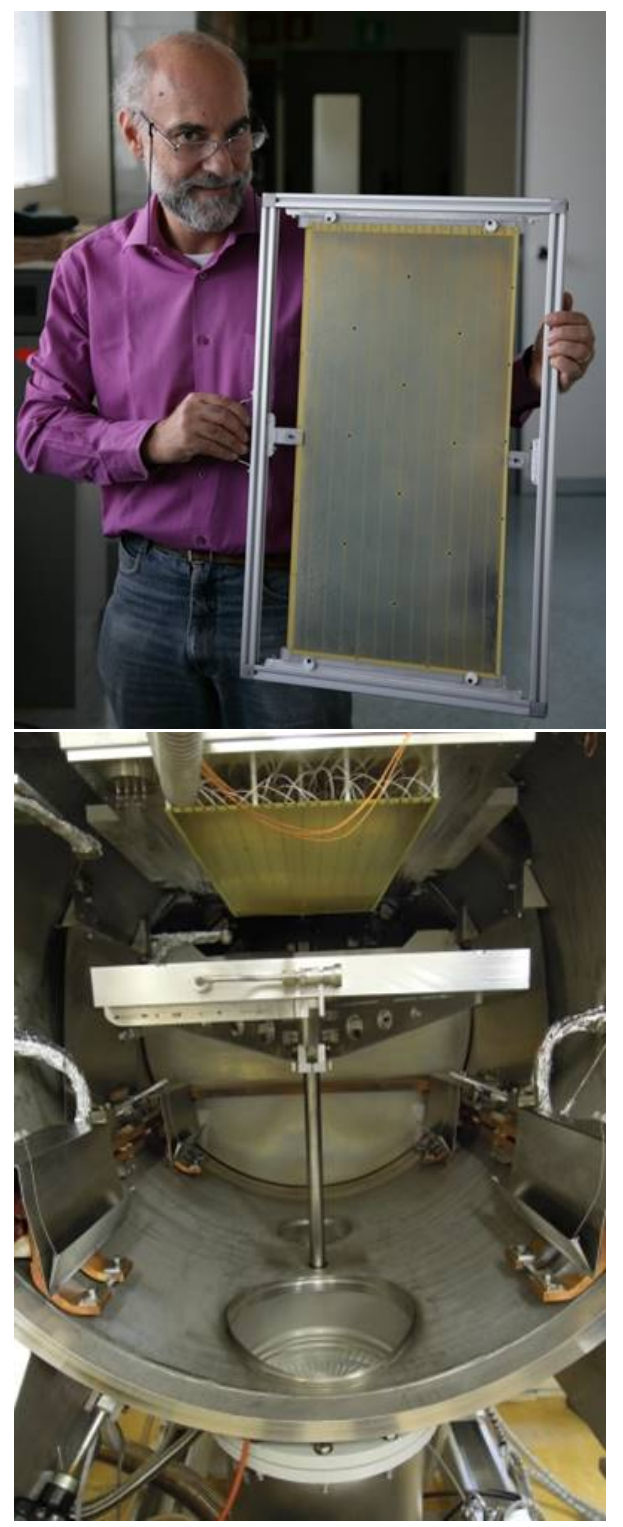

Figure 4. A THGEM in its handling frame (top) and inside the CsI deposition plant (bottom).

the entire THGEM area at a rate of $5 \mathrm{kHz} \mathrm{cm}^{-2}$, the readout being performed by the RD51 Scalable Readout System (SRS) based on the APV-25 analogue readout chip. This procedure is about 10 times faster and provides a detailed characterization of the local gain variations.

The validated and characterized THGEMs are transported to CERN for the Ni-Au coating process. A layer of about $5 \mu \mathrm{m}$ thickness of chemically deposited nickel and about $0.5 \mu \mathrm{m}$ chemically deposited gold are used as protection against copper oxidation and as substrate for the photoconverting CsI layer.

The procedure developed by the RD26 Collaboration for the photocathode production [6] is followed, whenever possible, for the CsI coating of the THGEMs faces to be used as reflective photocathodes. After cleaning and preparation the THGEM to be coated is mounted in a dedicated gas tight box with a two-pins electrical feed-through connection: the electrodes of the top layer of the THGEM 
are connected together via one pin and the electrodes of the bottom layer are connected via the other pin. The box is flushed with clean gas and used for THGEM storage but thanks to the electrical connections the THGEM can be biased and its electrical strength checked before and after the coating. The $300 \mathrm{~nm}$ thick CsI layer is deposited by evaporation using the dedicated CERN plant, with the typical conditions previously used for COMPASS and ALICE photocathode production (vavuum level around $10^{-6}$ mbar, $\mathrm{T} \approx 60{ }^{\circ} \mathrm{C}$ ). After the CsI evaporation a measurement of photocurrent is performed by illuminating with VUV light from a $\mathrm{D}_{2}$ lamp a spot of about $1 \mathrm{~cm}^{2}$ and recording the ratio between the THGEM photocurrent and the current from a reference PMT sensitive in the UV; the measurement is performed at 60 different points to map the response uniformity and it is repeated after lowering the temperature to $\approx 25^{\circ} \mathrm{C}$, before extracting the coated THGEM from the evaporation plant.

\section{The Micromegas and the other components}

COMPASS Micromegas are produced at CERN using the bulk technology (standard photolithographic lamination technique) to fix a stainless steel woven square micromesh (18 $\mu \mathrm{m}$ diameter wire, $63 \mu \mathrm{m}$ pitch, tensioned at $15 \mathrm{~N}$ ) onto an anode PCB specifically designed for COMPASS RICH-1 Micromegas. The anode PCB is $3.2 \mathrm{~mm}$ thick and has four copper layers: the anode layer is segmented in square pads of $8.0 \mathrm{~mm}$ pitch (with $0.5 \mathrm{~mm}$ inter-pad distance and pads of $7.5 \times 7.5 \mathrm{~mm}^{2} \mathrm{Cu}$ with $0.3 \mathrm{~mm}$ radius at the corners); a second layer, with the same segmentation, sits inside the PCB at $70 \mu \mathrm{m}$ from the anode, providing a capacitive coupling of $\approx 40 \mathrm{pF}$ between each anode pad an its facing (readout) pad, a value about 10 times larger than the capacitance between an anode pad and the micro-mesh; two layers are needed to route the paths from each readout pad to the corresponding pin of the connector for the front-end electronics and from each anode pad (trough a hole at the center of the readout pad) to the pin of the connector distributing the positive high voltage bias. Individual resistors of $470 \mathrm{M}$ Ohm are separating each anode pad from the high voltage power supply, to reduce the effects of a local discharge and let the non discharging pads unaffected by the discharge and recharge processes. The uniformity of the Micromegas amplification gap (128 $\mu \mathrm{m}$ nominal distance) is guaranteed by a square array of cylindrical micro-pillars with $300 \mu \mathrm{m} \mathrm{di-}$ ameter and $2 \mathrm{~mm}$ pitch (16 per pad) obtained from the photo-imageable polyimide film. A $5.5 \mathrm{~mm}$ wide coverlay border surrounding the detector's active area helps holding the micromesh and 24 circular (6 mm diameter) coverlay spots are prepared as bases for the THGEM fixation pillars. At the end of the production process the Micormegas is cut at its final $283 \times 586 \mathrm{~mm}^{2}$ dimension, readout connectors and bias resistor connectors are mounted and two identical Micromegas are glued onto the detector holder, side by side. The detector holder consists of an aluminum frame machined (by electro-erosion) from a 30 mm thick plate to provide stiffness, planarity and specific grooves for the insertion of the front-end boards, glued to a frame made of isolating material to avoid parasitic capacitance and machined to host the Micormegas. Strong Micromegas grounding is provided by embedding in conductive glue a large surface $\left(5 \times 260 \mathrm{~mm}^{2}\right)$ of each micromesh, on the side which is not in the active area of the final detector.

The gain uniformity of the Micromegas has been studied using a prototype detector, an $\mathrm{Ar} / \mathrm{CO}_{2}$ 70/30 gas mixture and a ${ }^{55} \mathrm{Fe}$ source: for an average gain of about 3000 the the local gain variations typically show a standard deviation of $5 \%$.

The micromesh, being the most extended unique electrode in the photon detector, is always kept at ground potential and in case of a discharge in the Micromegas the potential of the anode pad where the discharge takes place completely drops. To study the influence of such events on the neighboring pads, discharges have been systematically generated on a specific pad (by biasing it with an anomalously large potential) and the adjacent pads have been biased using for each pad a different high voltage channel from the power supply. The probability of correlated (within $\pm 100 \mathrm{~ns}$ ) discharges turned out to be negligible (no discharge cross-talk) when the adjacent pad was operated at normal voltage values, while raising the bias voltage of a neighboring pad led to more and more frequent correlated discharges, with a probability up to 0.7 when the same anomalous bias was applied, with a large majority of correlated events having a time difference $\leq 10$ ns.

After gluing the 48 THGEM pillars (24 for each Micromegas, M3 threaded, made of PEEK,) on the prepared coverlay bases and the high voltage distribution PCBs on the detector frame the hybrid PD is assembled: the gap between the Micromesh and the closer THGEM is $5 \mathrm{~mm}$ wide while the distance between the THGEMs is $3 \mathrm{~mm}$. The THGEMs are mounted in a staggered holes configuration (by $\mathrm{a} \approx 462 \mu \mathrm{m}$ displacement with respect to the aligned holes configuration) in order to favor the spread of the avalanche charges.

The electrostatic field above the photocathode is defined by two planes of wires $(100 \mu$ m diameter, $\mathrm{Cu}-\mathrm{Be}$ alloy with a flash of $\mathrm{Ni}$ and $\mathrm{Au}), 60 \mathrm{~cm}$ long, with a pitch of $4 \mathrm{~mm}$ and guard wires of $200 \mu \mathrm{m}$ diameter at the edges. The wires are manually soldered on half of a large $(645 \times$ $1400 \mathrm{~mm}^{2}$ ) mechanical frame which is also used, together with a similar size spacing frame, as holder of the existing Multi-Anode PMT based photon detectors. Special electrodes, embedded in the isolating material protections of the chamber frames are used to shape the field at the edges of the active volume, preventing large field values on any of the electrodes in the gas volume: the optimization of the final geometry required detailed numerical calculations of the fields taking into account all electrodes and their real shapes.

The preparation and mounting of the wire and spacing frames too requires several different steps since the overall mechanical structure of the detector is rather complex 
(see Fig. 1, right) and the procedures for assembling and installation of the new PDs are quite elaborated.

The high voltage distribution system provides independent bias values to each half of a THGEM face (6 sectors are connected to one HV channel via a resistive splitter with protection diodes to prevent HV drop cross-talk between sectors). Specific switch-on and safe-mode procedures prevent anomalous bias values to be applied to the THGEMs. The effective values of the applied bias will be continuously corrected to counteract the effects of environmental variables $(\mathrm{p}, \mathrm{T})$ variation: a gain stability in the order of $\pm 10 \%$ is expected, thanks to these corrections.

The signal from the readout pads are collected by front-end electronic cards [7] designed for COMPASS RICH-1: they host four APV25-S1 chips, each reading 108 pads. Three front-end cards are connected to a 10bit flash ADC digitizer board equipped with a FPGA performing on-line zero suppression. A cooling system using under-pressure water flow assures efficient removal of the heat produced by the readout.

The new hybrid PDs will be tested and installed on COMPASS RICH-1 before the 2016 run.

\section{Conclusions}

COMPASS RICH-1 will operate during the physics run of 2016 in an upgraded version with four new Photon Detectors based on hybrid THGEM + Micromegas technology. The expected high performance of the new PDs will allow to meet the strict requirements of the new COMPASS physics measurements. The production of all elements of the new PDs is almost complete and the procedures for test and validation of the components have been defined and applied. The recent developments of the field confirm the great potentialities of MPGD-based PDs and offer promising perspectives for their future applications.

\section{References}

[1] The COMPASS Collaboration, CERN/SPSC/2010014, SPSC-P-340, May 17, 2010; CERN/SPSC/2010022, SPSC-M-772, September 3, 2010.

[2] E.Albrecht et al., Nucl. Instr. and Meth. A 502 (2003) 112; E.Albrecht et al., Nucl. Instr. and Meth. A 502 (2003) 236; E.Albrecht et al., Nucl. Instr. and Meth. A 502 (2003) 266; E.Albrecht et al., Nucl. Instr. and Meth. A 510 (2003) 262; E.Albrecht et al., Nucl. Instr. and Meth. A 518 (2004) 586; M.Alekseev et al., Nucl. Instr. and Meth. A 553 (2005) 53; S.Costa et al., Nucl. Instr. and Meth. A 553 (2005) 135; E.Albrecht et al., Nucl. Instr. and Meth. A 553 (2005) 215; P.Abbon et al., Czech. J. Phys. 56, Suppl. F (2006) 307; P.Abbon et al., Czech. J. Phys. 56, Suppl. F (2006) 315; P.Abbon et al., Czech. J. Phys. 56 Suppl. F (2006) 323; P.Abbon et al., Nucl. Instr. and Meth. A 567 (2007) 114; P.Abbon et al., Nucl. Instr. and Meth. A 572 (2007) 419; P.Abbon et al., Nucl. Instr. and Meth. A 580 (2007) 906; P.Abbon et al., Nucl. Instr. and Meth. A 581 (2007) 419; P.Abbon et al., Nucl. Instr. and Meth. A 587 (2008) 371; P.Abbon et al., Nucl. Instr. and Meth. A 595 (2008) 23; P.Abbon et al., Nucl. Instr. and Meth. A 595 (2008) 177; M.Alexeev et al., Nucl. Instr. and Meth. A 595 (2008) 194; P.Abbon et al., Nucl. Instr. and Meth. A 595 (2008) 204; S.Dalla Torre et al., Nucl. Instr. and Meth. A 595 (2008) 220; P.Abbon et al., Nucl. Instr. and Meth. A 595 (2008) 233; P.Abbon et al., Nucl. Instr. and Meth. A 616 (2010) 21; P.Abbon et al., Nucl. Instr. and Meth. A 623 (2010) 330; P.Abbon et al., Nucl. Instr. and Meth. A 631 (2011) 26; P.Abbon et al., Nucl. Instr. and Meth. A 639 (2011) 15; M.Alexeev et al., Nucl. Instr. and Meth. A 639 (2011) 219; S.Dalla Torre et al., Nucl. Instr. and Meth. A 639 (2011) 271; F.Tessarotto et al., JINST 9 (2014) C09011; M.Alexeev et al., Nucl. Instr. and Meth. A 766 (2014) 208.

[3] M.Alexeev et al., JINST 9 (2014) P01006; M.Alexeev et al., Nucl. Instr. and Meth. A 766 (2014) 199.

[4] M.Alexeev et al., Nucl. Instr. and Meth. A 610 (2009) 174; M.Alexeev et al., Nucl. Instr. and Meth. A 617 (2010) 396; M.Alexeev et al., Nucl. Instr. and Meth. A 623 (2010) 129; M.Alexeev et al., JINST 5 (2010) P03009; M.Alexeev et al., Nucl. Instr. and Meth. A 639 (2011) 130; M.Alexeev et al., JINST 7 (2012) C02014; M.Alexeev et al., Nucl. Instr. and Meth. A 695 (2012) 159; M.Alexeev et al., Physics Procedia 37 (2012) 781; M.Alexeev et al., Nucl. Instr. and Meth. A 732 (2013) 264; M.Alexeev et al., JINST 9 (2014) C03046; M.Alexeev et al., JINST 9 (2014) C09017; M.Alexeev et al., JINST 10 (2014) P03026;

[5] M.Alexeev et al., Nucl. Instr. and Meth. A 766 (2014) 133.

[6] A.Braem et al., Nucl. Instr. and Meth. A 502 (2003) 205.

[7] P.Abbon et al., Nucl. Instr. and Meth. A 567 (2006) 104. 